\title{
Nitrate and nitrogen oxides: Sources, health effects and their remediation
}

\begin{abstract}
Increased use of nitrogenous $(\mathrm{N})$ fertilizers in agriculture has significantly altered the global $\mathrm{N}$-cycle because they release nitrogenous gases of environmental concerns. The emission of nitrous oxide (N2O) contributes to the global greenhouse gas accumulation and the stratospheric ozone depletion. In addition, it causes nitrate leaching problem deteriorating ground water quality. The nitrate toxicity has been reported in a number of studies showing the health hazards like methemoglobinemia in infants and is a potent cause of cancer. Despite these evident negative environmental as well as health impacts, consumption of $\mathrm{N}$ fertilizer cannot be reduced in view of the food security for the teeming growing world population. Various agronomic and genetic modifications have been practiced to tackle this problem. Some agronomic techniques adopted include split application of $\mathrm{N}$, use of slow-release fertilizers, nitrification inhibitors and encouraging the use of organic manure over chemical fertilizers. As a matter of fact, the use of chemical means to remediate nitrate from the environment is very difficult and costly. Particularly, removal of nitrate from water is difficult task because it is chemically non-reactive in dilute aqueous solutions. Hence, the use of biological means for nitrate remediation offers a promising strategy to minimize the ill effects of nitrates and nitrites. One of the important goals to reduce N-fertilizer application can be effectively achieved by choosing $\mathrm{N}$-efficient genotypes. This will ensure the optimum uptake of applied $\mathrm{N}$ in a balanced manner and exploring the molecular mechanisms for their uptake as well as metabolism in assimilatory pathways. The objectives of this paper are to evaluate the interrelations which exist in the terrestrial ecosystems between the plant type and characteristics of nutrient uptake and analyze the global consumption and demand for fertilizer nitrogen in relation to cereal production, evaluate the various methods used to determine nitrogen use efficincy (NUE), determine NUE for the major cereals grown across large agroclimatic regions, determine the key factors that control NUE, and finally analyze various strategies available to improve the use efficiency of fertilizer nitrogen.
\end{abstract}

Keyword: Environment; Phytoremediation; Nitrate pollution; Nitrogen oxides; Ozone depletion; Nitrogen use efficiency; Nitrate reductase; Nitrite reductase; Glutamine synthase; Nitrogen emission; Nitrous oxide; Environmental pollution; N-efficient genotypes; Methemoglobinemia; Transcription factors; Nitrogen metabolism pathway; Nitrate toxicity; Slow-release fertilizers; Nitrate uptake; Nitrate assimilation; Transgenic plants; Global warming; Fertilizer use efficiency; Nitrosamines; Global climate change 